\title{
Paeoniflorin inhibits doxorubicin-induced cardiomyocyte apoptosis by downregulating microRNA-1 expression
}

\author{
JIAN-ZHE LI ${ }^{1 *}$, XIU-NENG TANG ${ }^{1 *}$, TING-TING LI² ${ }^{2}$ LI-JUAN LIU ${ }^{3}$, SHU-YI YU ${ }^{4}$, \\ GUANG-YU ZHOU ${ }^{1}$, QING-RUI SHAO ${ }^{1}$, HUI-PING SUN ${ }^{5}, \mathrm{CHENG} \mathrm{WU}^{1}$ and YANG YANG ${ }^{1}$ \\ ${ }^{1}$ Department of Pharmacy, Ruikang Hospital, Guangxi University of Chinese Medicine, Nanning, Guangxi 530011; \\ ${ }^{2}$ Department of Pharmacy, People's Hospital of Xishuangbanna Dai Autonomous Prefecture, Jinghong, Yunnan 666100; \\ ${ }^{3}$ Department of Pharmacy, Jiangxi Provincial Cancer Hospital, Nanchang, Jiangxi 330029; ${ }^{4}$ Advanced Research Center, \\ Central South University, Changsha, Hunan 410078; ${ }^{5}$ Department of Anesthesia, Hunan Cancer Hospital, \\ The Affiliated Cancer Hospital of Xiangya School of Medicine, Central South University, \\ Changsha, Hunan 410006, P.R. China
}

Received January 26, 2015; Accepted March 8, 2016

DOI: $10.3892 / \mathrm{etm} .2016 .3182$

\begin{abstract}
Doxorubicin (DOX) is an effective anthracycline anti-tumor antibiotic. Because of its cardiotoxicity, the clinical application of DOX is limited. Paeoniflorin (PEF), a monoterpene glucoside extracted from the dry root of Paeonia, is reported to exert multiple beneficial effects on the cardiovascular system. The present study was designed to explore the protective effect of PEF against DOX-induced cardiomyocyte apoptosis and the underlying mechanism. In cultured $\mathrm{H} 9 \mathrm{c} 2$ cells, PEF $(100 \mu \mathrm{mol} / \mathrm{l})$ was added for $2 \mathrm{~h}$ prior to exposure to $\operatorname{DOX}(5 \mu \mathrm{mol} / \mathrm{l})$ for $24 \mathrm{~h}$. Cell viability, creatine kinase activity, cardiomyocyte apoptosis, intracellular reactive oxygen species (ROS) levels, and the expression of microRNA-1 (miR-1) and B-cell lymphoma 2 (Bcl-2) were measured following treatment with PEF and/or DOX. The results showed that treatment with DOX notably induced cardiomyocyte apoptosis, concomitantly with enhanced ROS generation, upregulated miR-1 expression and downregulated Bcl-2 expression. These effects of DOX were significantly inhibited by pretreatment of the cells with PEF. These results suggest that the inhibitory effect of PEF
\end{abstract}

Correspondence to: Dr Li-Juan Liu, Department of Pharmacy, Jiangxi Provincial Cancer Hospital, 519 East Beijing Road, Nanchang, Jiangxi 330029, P.R. China

E-mail: 1ijuan_liu0791@126.com

Professor Shu-Yi Yu, Advanced Research Center, Central South University, 110 Xiang-Ya Road, Changsha, Hunan 410078, P.R. China

E-mail: shuyi_yu0731@126.com

${ }^{*}$ Contributed equally

Key words: cardiomyocyte apoptosis, doxorubicin, microRNA-1, paeoniflorin, reactive oxygen species on DOX-induced cardiomyocyte apoptosis may be associated with downregulation of miR-1 expression via a reduction in ROS generation.

\section{Introduction}

Doxorubicin (DOX) is an effective anthracycline antitumor antibiotic and is extensively used for treatment of numerous types of malignant tumors, including osteosarcoma, breast carcinoma and lung cancer (1-3). However, because of cumulative and dose-dependent cardiotoxicity, the clinical application of DOX is limited. Although the exact physiopathological mechanism of DOX-induced cardiotoxicity is not yet understood, cardiomyocyte apoptosis is acknowledged to play an important role in DOX-associated cardiotoxicity $(4,5)$.

MicroRNAs (miRs) are a class of endogenous non-coding RNAs of 22 nucleotides, which participate in the regulation of protein-coding gene expression by binding to the 3'-untranslated regions of target mRNA at the post-transcriptional level (6). It has been predicted that there are $>1,000 \mathrm{miRs}$ in the human genome, and miRs are estimated to regulate as many as $60 \%$ of all human mRNAs $(7,8)$. It is well recognized that miRs play important roles in a large variety of physiological and pathological processes, such as embryonic development, tumor formation and modulation of the inflammatory response (9-11). Among the known miRs, miR-1 is a muscle-enriched miR and highly expressed in the myocardium (12). Therefore, aberrant expression of miR-1 is closely associated with myocardial diseases. It has been reported that miR-1 expression is markedly increased in ischemia/reperfusion (IR) or hypoxia/reoxygenation-induced cardiac myocyte apoptosis (13). In addition, in miR-1 transgenic mice, cardiac myocyte apoptosis was found to be exacerbated compared with that in wild-type mice when subjected to myocardial IR injury; by contrast, knockdown of miR-1 using a locked nucleic acid-modified oligonucleotide against miR-1 significantly attenuated IR-induced cardiac myocyte apoptosis (14). However, the role of miR-1 in DOX-induced cardiomyocyte apoptosis is not understood. 
Paeoniflorin (PEF), a monoterpene glucoside, is the major active ingredient of Paeonia lactiflora Pall. (family Paeoniaceae), which is a traditional Chinese herbal medicine that has been used in China for $>1,000$ years. It has been reported that PEF exerts multiple pharmacological activities, such as inhibition of tumor invasion and metastasis (15), reduction of inflammatory factor production (16), prevention of insulin resistance (17) and neuroprotective effects (18). Our previous study demonstrated that PEF was able to inhibit DOX-induced cardiomyocyte apoptosis by reducing the production of reactive oxygen species (ROS) (19); however, the downstream mechanism remains unclear.

In the present study, the role of miR-1 in DOX-induced cell apoptosis was explored using a cultured H9c2 rat cardiomyocyte cell line, and whether the anti-apoptotic effect of PEF was related to a regulatory effect on miR-1 expression was investigated.

\section{Materials and methods}

Materials. The H9c2 cell line was acquired from Academia Sinica (Shanghai, China). PEF was obtained from Yangling Dongke Maidisen Pharmaceutical Co., Ltd. (Xi'an, China). DOX was purchased from Sigma-Aldrich (St. Louis, MO, USA). Dulbecco's modified Eagle's medium (DMEM) and fetal bovine serum (FBS) were from Gibco (Thermo Fisher Scientific, Inc., Waltham, MA, USA). mirVana miR isolation kits and TaqMan ${ }^{\mathrm{TM}}$ miR Reverse Transcription kits were purchased from Applied Biosystems (Thermo Fisher Scientific, Inc.). B-cell lymphoma 2 (Bcl-2; ab59348) and $\beta$-actin (ab1801) antibodies were from Abcam (Cambridge, UK). The following were purchased from Beyotime Institute of Biotechnology (Jiangsu, China): 3-(4,5-Dimethylthiazol-2-yl)-2,5-diphenyl tetrazolium bromide (MTT), Hoechst 33342 fluorescent dye and reactive oxygen species (ROS) detection kit. The creatine kinase $(\mathrm{CK})$ assay kit was acquired from Nanjing Jiancheng Bioengineering Institute (Nanjing, China).

Cell culture and treatment. H9c2 cells were cultured in DMEM containing $15 \%(\mathrm{v} / \mathrm{v})$ FBS. The medium was supplemented with $100 \mathrm{U} / \mathrm{ml}$ penicillin (Guangzhou Baiyunshan Tianxin Pharmaceutical Co., Ltd., Guangdong, China) and $100 \mu \mathrm{g} / \mathrm{ml}$ streptomycin (Harbin Pharmaceutical Group Holding Co., Ltd., Heilongjiang, China). All cells were grown under a humidified atmosphere of $5 \% \mathrm{CO}_{2}$ at $37^{\circ} \mathrm{C}$. The medium was changed every 2-3 days. When cells reached $80 \%$ confluence, they were made quiescent by serum starvation $(0.5 \% \mathrm{FBS})$ for $24 \mathrm{~h}$, and then the cells were randomly allocated to 4 groups: i) The control group (cultured in normal condition); ii) the DOX group (incubated with $5 \mu \mathrm{mol} / 1 \mathrm{DOX}$ for $24 \mathrm{~h}$ ); iii) the $\mathrm{PEF}+\mathrm{DOX}$ group (cells were treated with $100 \mu \mathrm{mol} / 1 \mathrm{PEF}$ for $2 \mathrm{~h}$ prior to exposure to $5 \mu \mathrm{mol} / 1 \mathrm{DOX}$ for $24 \mathrm{~h}$ ); and iv) the PEF group (incubated with $100 \mu \mathrm{mol} / 1 \mathrm{PEF}$ for $26 \mathrm{~h}$ ).

Evaluation of cell injury. Cell viability was assessed by MTT assay. Briefly, following DOX treatment, the medium was aspirated, and the cells were washed twice with PBS, then $0.5 \mathrm{mg} / \mathrm{ml} \mathrm{MTT} \mathrm{solution} \mathrm{was} \mathrm{added} \mathrm{to} \mathrm{each} \mathrm{well} \mathrm{and} \mathrm{the} \mathrm{plate}$ was incubated for $4 \mathrm{~h}$ at $37^{\circ} \mathrm{C}$ in an atmosphere of $5 \% \mathrm{CO}_{2}$. After that, $100 \mu 1$ dimethysulfoxide (DMSO) was added to dissolve formazan. The absorbance was read at $490 \mathrm{~nm}$ using a microplate reader (Molecular Devices, Sunnyvale, CA, USA). Cell viability was expressed as a percentage of the control. The activity of CK, an indicator of cardiomyocyte injury, was detected using a commercially available colorimetric assay kit according to the manufacturer's protocol.

Measurement of cardiomyocyte apoptosis. Apoptotic cells were detected by Hoechst 33342 fluorescent dye using an inverted fluorescence microscope (Nikon Eclipse TE-2000; Nikon Corporation, Tokyo, Japan). Briefly, after DOX treatment, the medium was aspirated, and the cells were washed twice with PBS. After that, cells were incubated with Hoechst $33342(0.1 \mathrm{mg} / \mathrm{ml})$ in the dark at $37^{\circ} \mathrm{C}$ for $20 \mathrm{~min}$. Hoechst-stained nuclei were detected by inverted fluorescence microscopy at an emission wavelength of $521 \mathrm{~nm}$. The data are expressed as a percentage of apoptotic cells to total cells.

Determination of intracellular ROS. The levels of intracellular ROS were measured using 2',7'-dichlorofluorescein diacetate, a well characterized probe. Briefly, following DOX treatment, the medium was aspirated and cells were washed three times with serum-free DMEM. After that, cells were incubated with 2',7'-dichlorofluorescein diacetate at a final concentration of $10 \mu \mathrm{M}$ in the dark at $37^{\circ} \mathrm{C}$ for $20 \mathrm{~min}$. The fluorescence intensity was measured using a fluorospectrophotometer (Shimadzu Corporation, Kyoto, Japan) at an excitation wavelength at $488 \mathrm{~nm}$ and an emission wavelength at $525 \mathrm{~nm}$. The data are expressed as a percentage of the control.

\section{Quantitative polymerase chain reaction ( $q P C R)$}

miR-1 expression. Following treatment, total RNA was isolated from $\mathrm{H} 9 \mathrm{c} 2$ cells using the mirVana miR isolation kit according to the manufacturer's protocol. cDNA was synthesized from $1 \mu \mathrm{g}$ total RNA using the TaqMan ${ }^{\mathrm{TM}}$ miR reverse transcription kit (Promega Corporation, Madison, WI, USA). cDNA was diluted 15-fold and then used in PCR reactions. Reactions contained specific primers for miR-1 or U6; the latter was used as an internal control. The primer sequences were as follows: miR-1 forward, 5'-ACACTCCAGCTGGGG TGTGGAATGTA-3' and reverse, 5'-TGGTGTCGTGGA GTCG-3; U6 forward, 5'-ACACTCCAGCTGGGGTGCTCG CTTCGGCAGCACA-3' and reverse, 5'-AGGGTCCGAGGT ATTC-3'. PCR was performed using TaqMan universal PCR master mix (Applied Biosystems; Thermo Fisher Scientific, Inc.) with the following conditions: $10 \mathrm{~min}$ at $95^{\circ} \mathrm{C}$ followed by 40 cycles of $95^{\circ} \mathrm{C}$ for $15 \mathrm{sec}$ and $60^{\circ} \mathrm{C}$ for $1 \mathrm{~min}$. All amplification reaction for each sample was carried out four times. The relative expression values were normalized to the expression of U6 using the $2^{-\Delta \Delta \mathrm{Cq}}$ method (20).

Bcl-2 mRNA expression. Following treatment, total RNA was isolated using TRIzol reagent (Invitrogen; Thermo Fisher Scientific, Inc.) and quantified by measuring the optical density at $260 \mathrm{~nm} .1 \mu \mathrm{g}$ RNA from each sample was reverse-transcribed to cDNA using an M-MLV reverse transcriptase kit, and then cDNA was used for qPCR. Bcl-2 mRNA expression was quantitatively analyzed using an Applied Biosystems 7300 Real-Time PCR system with a Power SYBR Green PCR Master Mix kit (both Thermo Fisher Scientific, Inc.). PCR primers were as follows: Bcl-2 primers: forward, 5'-CCGGGA 
A

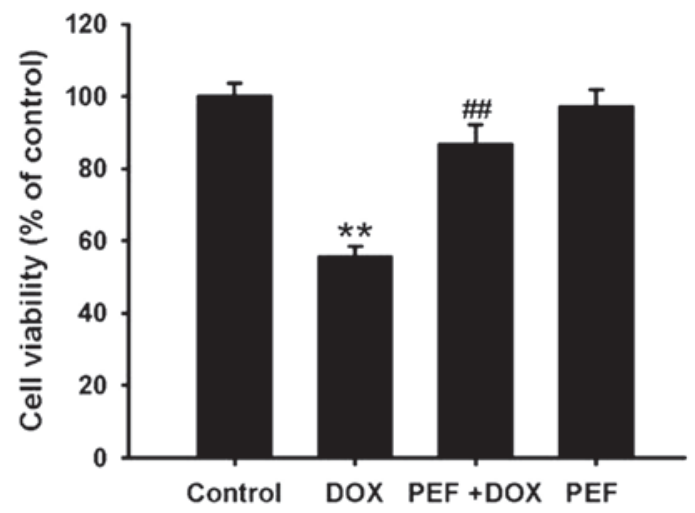

B

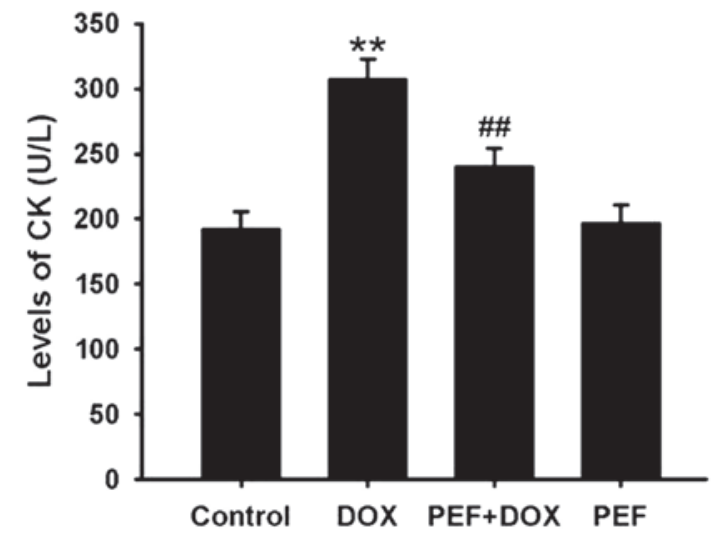

Figure 1. Effect of PEF on (A) cell viability and (B) levels of CK induced by DOX in H9c2 cells. Data are expressed as the mean \pm standard error of the mean and represent four independent experiments. ${ }^{* *} \mathrm{P}<0.01$ vs. the control group, ${ }^{\# \#} \mathrm{P}<0.01$ vs. the DOX group. PEF, paeoniflorin; DOX, doxorubicin; $\mathrm{CK}$, creatine kinase.

GAACAGGGTATGATAA-3' and reverse, 5'-CCCACTCGT AGCCCCTCTG-3'; glyceraldehyde 3-phosphate dehydrogenase (GAPDH) primers: forward, 5'-TGGCCTCCAAGGAG TAAGAAAC-3' and reverse, 5'-GGCCTCTCTCTTGCTCTC AGTATC-3'. The PCR amplification program involved initial denaturation at $95^{\circ} \mathrm{C}$ for $10 \mathrm{~min}$, followed by 40 cycles of denaturation at $95^{\circ} \mathrm{C}$ for $15 \mathrm{sec}$ and annealing at $60^{\circ} \mathrm{C}$ for $60 \mathrm{sec}$. All amplification reactions for each sample were carried out four times, and the relative expression values were normalized to the expression value of GAPDH using the $2^{-\Delta \Delta \mathrm{Cq}}$ method (20).

Western blot analysis. The protein expression of Bcl-2 was detected by western blot analysis. Briefly, following the various treatments, cells were lysed with ice-cold lysis buffer, and the protein concentration was measured using a Bradford protein assay (Beyotime Institute of Biotechnology, Inc.). Equal amounts of protein were separated by $10 \%$ sodium dodecyl sulfate-polyacrylamide gel electrophoresis and transferred to a nitrocellulose membrane. After transferring the protein samples, the membranes were blocked using $5 \%$ milk powder in Tris-buffered saline and Tween 20 at room temperature for $1 \mathrm{~h}$ and then incubated with primary rabbit polyclonal antibodies for Bcl-2 and $\beta$-actin (both 1:1,000 dilution) overnight at $4^{\circ} \mathrm{C}$, followed by incubation with the corresponding goat anti-rabbit horseradish peroxidase-conjugated secondary antibody (1:5,000 dilution; ab175773; Abcam) for $1 \mathrm{~h}$ at room temperature. The protein bands were scanned and densitometrically analyzed using an automatic image analysis system (Alpha Innotech Corporation, San Loandra, CA, USA), and the results were normalized to $\beta$-actin expression.

Statistical analysis. Data are presented as means \pm standard error of the mean. All values were analyzed using analysis of variance and the Newman-Keuls Student's t-test. $\mathrm{P}<0.05$ was considered to indicate a statistically significant difference.

\section{Results}

Effect of PEF on DOX-induced cell injury. The MTT assay showed that cell viability was clearly decreased following DOX treatment, and this reduction in viability was significantly attenuated by pretreatment with PEF for $2 \mathrm{~h}(\mathrm{P}<0.01$; Fig. 1A). In line with the MTT assay results, the levels of CK were markedly increased following incubation with DOX, and this effect of DOX was also significantly inhibited by pretreatment with PEF ( $\mathrm{P}<0.01$; Fig. 1B). However, PEF alone had no effect on cell viability or the levels of CK.

Effect of PEF on DOX-induced apoptosis. Cardiomyocyte apoptosis was measured by Hoechst 33342 staining. The results showed that DOX treatment notably increased the ratio of apoptotic cells compared with that in the control group. However, pretreatment with PEF for $2 \mathrm{~h}$ significantly inhibited DOX-induced cell apoptosis ( $\mathrm{P}<0.01 ;$ Fig. 2). PEF alone had no effect on cardiomyocyte apoptosis.

Effect of PEF on DOX-induced intracellular ROS production. The levels of intracellular ROS, indicated by the 2',7'-dichloroflurorescein fluorescence intensity, were notably increased in DOX-treated cells compared with control cells. However, pretreatment with PEF for $2 \mathrm{~h}$ significantly suppressed the intracellular ROS production induced by DOX $(\mathrm{P}<0.01$; Fig. 3). PEF alone had no such effect.

Effect of PEF on DOX-induced miR-1 expression. miR-1 expression was markedly upregulated after DOX treatment compared with that in the control group. This effect of DOX was significantly inhibited by pretreatment with PEF for $2 \mathrm{~h}$ $(\mathrm{P}<0.01$; Fig. 4). PEF alone had no effect on miR-1 expression.

Effect of PEF on Bcl-2 expression. PCR analysis showed that the mRNA expression of Bcl-2, an anti-apoptotic gene, was significantly downregulated in DOX-treated cells $(\mathrm{P}<0.01)$. Consistent with these results, Bcl-2 protein expression was also markedly decreased after incubation with DOX $(\mathrm{P}<0.01)$. However, these effects induced by DOX were inhibited by pretreatment with $\mathrm{PEF}$ for $2 \mathrm{~h}(\mathrm{P}<0.01$ and $\mathrm{P}<0.05$, respectively; Fig. 5). PEF alone had no effect on Bcl-2 expression.

\section{Discussion}

In the current study, it was found that DOX-induced cardiomyocyte apoptosis was accompanied by increased intracellular ROS production, upregulated miR-1 expression and downregulated Bcl-2 mRNA and protein expression. These effects of DOX were markedly inhibited by pretreatment with PEF. 
A

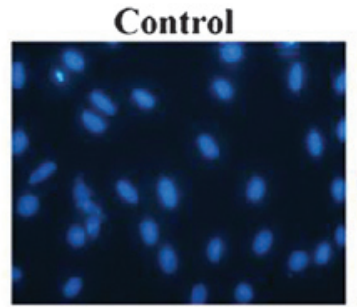

PEF+DOX

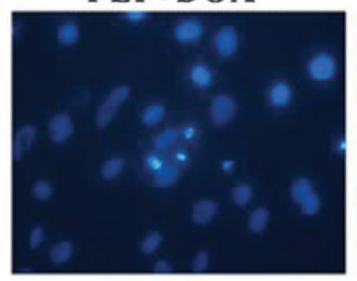

B

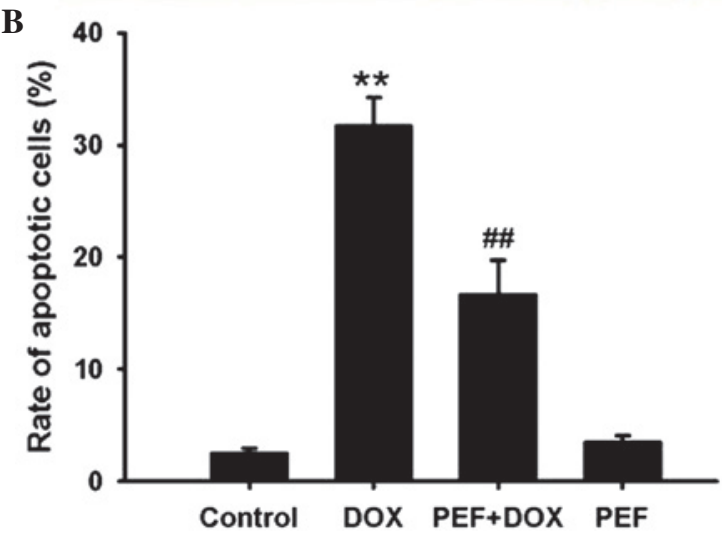

Figure 2. Effect of PEF on apoptosis induced by DOX in H9c2 cells. (A) representative fluorescence microscopy images of Hoechst 33342 staining (magnification, x200). (B) rate of apoptotic cells. Data are expressed as the mean \pm standard error of the mean and represent four independent experiments. ${ }^{* *} \mathrm{P}<0.01$ vs. the control group, ${ }^{\# \#} \mathrm{P}<0.01$ vs. the DOX group. PEF, paeoniflorin; DOX, doxorubicin.

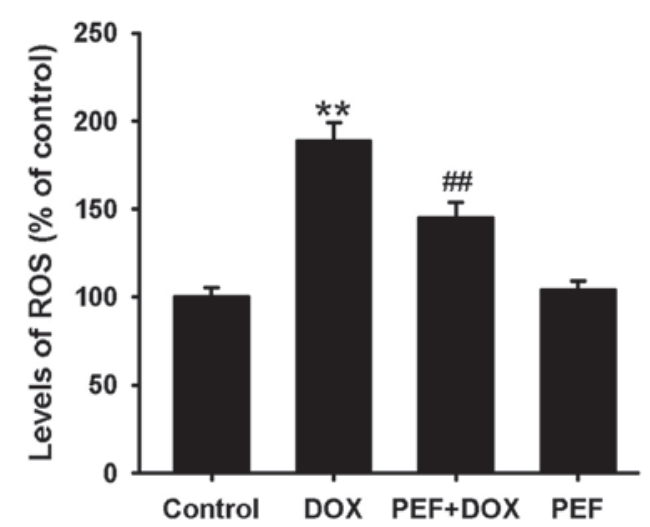

Figure 3. Effect of PEF on ROS production induced by DOX in H9c2 cells. Data are expressed as the mean \pm standard error of the mean and represent four independent experiments. ${ }^{* *} \mathrm{P}<0.01$ vs. the control group, ${ }^{\# \#} \mathrm{P}<0.01$ vs the DOX group. PEF, paeoniflorin; DOX, doxorubicin; ROS, reactive oxygen species.

These results indicate that the anti-apoptotic effect of PEF was associated with a reduction in ROS production and downregulation of miR-1 expression.

It is acknowledged that cardiomyocyte apoptosis plays an important role in DOX-induced cardiotoxicity $(4,5)$. However,

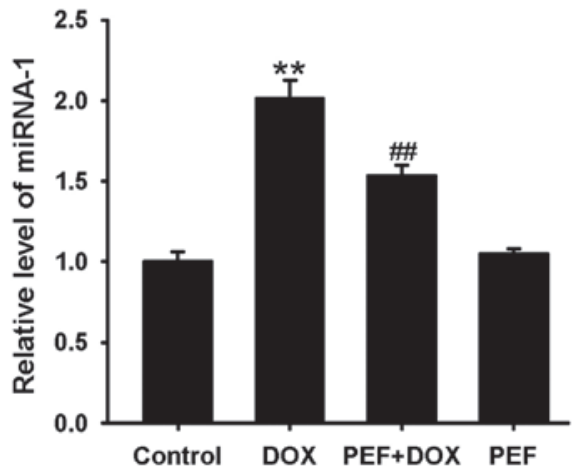

Figure 4. Effect of PEF on miRNA-1 expression induced by DOX in H9c2 cells. Data are expressed as the mean \pm standard error of the mean and represent four independent experiments. ${ }^{* *} \mathrm{P}<0.01$ vs. the control group, ${ }^{\# \#} \mathrm{P}<0.01$ vs. the DOX group. PEF, paeoniflorin; DOX, doxorubicin.

A

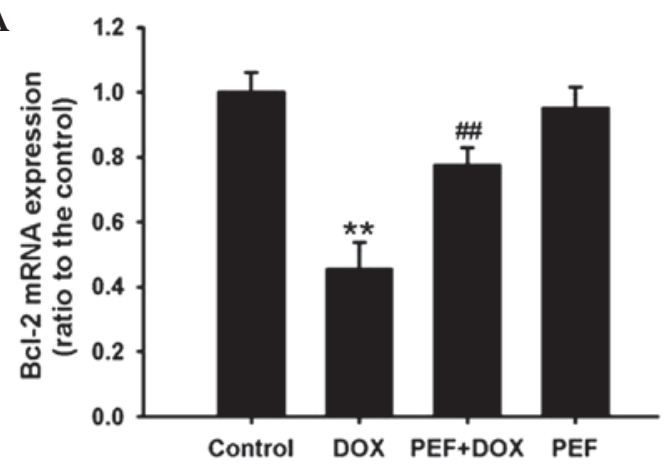

B

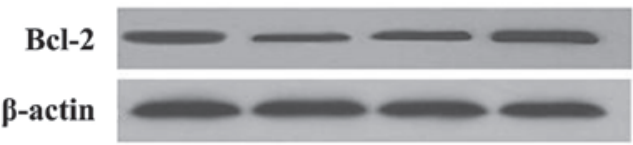

C

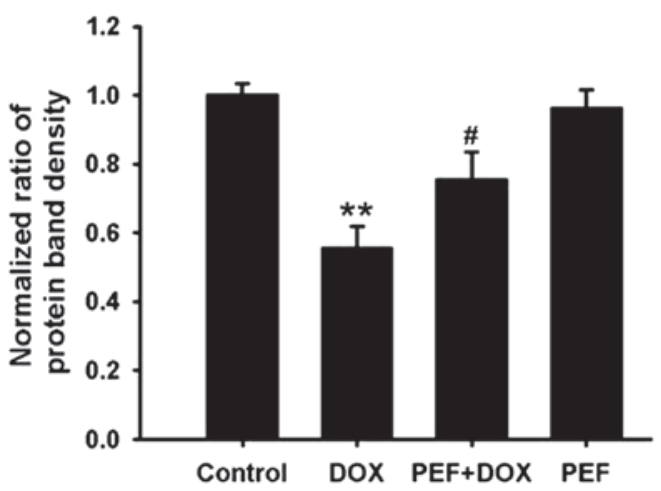

Figure 5. Effect of PEF on Bcl-2 expression induced by DOX in H9c2 cells. (A) Bcl-2 mRNA expression determined by quantitative polymerase chain reaction; (B) representative image of $\mathrm{Bcl}-2$ protein expression analyzed by western blot; (C) optical density of protein bands. Data are expressed as the mean \pm standard error of the mean and represent four independent experiments. ${ }^{* *} \mathrm{P}<0.01$ vs. control group, ${ }^{\#} \mathrm{P}<0.05,{ }^{\# \#} \mathrm{P}<0.01$ vs. DOX group. $\mathrm{PEF}$, paeoniflorin; DOX, doxorubicin; Bcl-2, B-cell lymphoma 2.

the exact mechanism of DOX-induced cardiomyocyte apoptosis is not fully understood. miR-1 is a muscle-specific miR and highly expressed in the myocardium (12). The abnormal expression of miR-1 is involved in a number of heart diseases. It has been reported that changes in miR-1 expression are associated with, for example, arrhythmia, myocardial infarction, 
cardiac remodeling and heart failure (21). There is evidence that upregulated miR-1 expression is closely associated with cardiomyocyte apoptosis; in models of IR-, high glucose- or $\mathrm{H}_{2} \mathrm{O}_{2}$-induced cardiomyocyte apoptosis, miR-1 expression has been shown to be significantly increased $(13,22,23)$. Further studies have found that the level of miR-1 is inversely correlated with the expression of Bcl-2, which is an anti-apoptotic protein that has been demonstrated to be a potential target of miR-1 $(13,23)$. Overexpression of miR-1 has been shown to exacerbate cardiomyocyte apoptosis induced by IR in mouse models, while knockdown of miR-1 expression significantly attenuated cardiac injury (14). The aforementioned studies suggest that upregulated expression of miR-1 plays a critical role in cardiomyocyte apoptosis via the post-transcriptional repression of $\mathrm{Bcl}-2$ expression, and reducing miR-1 expression may be a promising strategy for the prevention of cardiomyocyte apoptosis.

The mechanism by which miR expression is regulated is not fully clear. A number of factors, including ultraviolet radiation and tertiary-butyl hydroperoxide can lead to changes in the expression of multiple miRs, which is closely associated with increased ROS production (24,25). Schmelzer et al (26) have demonstrated that bacterial lipopolysaccharide induces miR-146a expression via a ROS-dependent pathway as this effect was inhibited by pretreatment with the antioxidant ubiquinol-10. Furthermore, the expression of multiple miRs, including miR-1, was found to change after treatment with $\mathrm{H}_{2} \mathrm{O}_{2}$ (27,28). These studies suggest that miR expression may be modulated by ROS. A number of studies have demonstrated that DOX-induced cardiomyocyte apoptosis is closely associated with excessive ROS production $(29,30)$. Therefore, we hypothesized that DOX-induced cardiomyocyte apoptosis may be associated with upregulation of miR-1 expression by an increase in ROS production. In the present study, it was indeed found that DOX significantly increased ROS generation concomitantly with upregulation of miR-1 expression. Thus, reduction of ROS production may be an effective method for inhibiting DOX-induced cardiomyocyte apoptosis via the downregulation of miR-1 expression.

PEF, a monoterpene glucoside extracted from the dry root of Paeonia lactiflora, has been reported to exert multiple pharmacological activities (15-18). Studies have suggested that PEF has anti-oxidative activity (19,31). Furthermore, our previous study demonstrated that PEF was able to inhibit DOX-induced cardiomyocyte apoptosis by reducing ROS production (19), but the downstream mechanism was not clear. Since miR-1 expression is regulated by ROS, and PEF has an anti-oxidative property, we speculated that the anti-apoptotic effect of PEF may be associated with downregulation of miR-1 expression via inhibition of ROS production. In the present study, it was confirmed that PEF markedly inhibited ROS generation, which was accompanied by downregulation of miR-1 expression.

In summary, the results of the current study suggest that the upregulation of miR-1 expression plays an important role in DOX-induced cardiomyocyte apoptosis by the post-transcriptional repression of $\mathrm{Bcl}-2$ expression. Furthermore, the inhibitory effect PEF against DOX-induced cardiomyocyte apoptosis may be associated with the downregulation of miR-1 expression via a reduction in ROS production.

\section{Acknowledgements}

This study was supported by the National Nature Science Foundation of China (grant no. 81460613 to J.Z. Li, 81101476 to S.Y. Yu and 81260337 to L.J. Liu), the Guangxi Nature Science Foundation of China (grant no. 2013GXNSFBA019126 to J.Z. Li], the Bureau of Public Health of Guangxi Province (grant no. Z2013206) and the Guangxi Administration of Traditional Chinese Medicine (grant no. GZZC14-36).

\section{References}

1. Tacar O, Indumathy S, Tan ML, Baindur-Hudson S, Friedhuber AM and Dass CR: Cardiomyocyte apoptosis vs autophagy with prolonged doxorubicin treatment: Comparison with osteosarcoma cells. J Pharm Pharmacol 67: 231-243, 2015.

2. Rastegar H, Ahmadi Ashtiani H, Anjarani S, Bokaee S, Khaki A and Javadi L: The role of milk thistle extract in breast carcinoma cell line (MCF-7) apoptosis with doxorubicin. Acta Med Iran 51: 591-598, 2013.

3. Shi X, Li C, Gao S, Zhang L, Han H, Zhang J, Shi W and Li Q: Combination of doxorubicin-based chemotherapy and polyethylenimine/p53 gene therapy for the treatment of lung cancer using porous PLGA microparticles. Colloids Surf B Biointerfaces 122: 498-504, 2014.

4. Gao S, Li H, Cai Y, Ye JT, Liu ZP, Lu J, Huang XY, Feng XJ, Gao H, Chen SR, et al: Mitochondrial binding of $\alpha$-enolase stabilizes mitochondrial membrane: Its role in doxorubicin-induced cardiomyocyte apoptosis. Arch Biochem Biophys 542: 46-55, 2014.

5. Li D, Li J, An Y, Yang Y and Zhang SQ: Doxorubicin-induced apoptosis in $\mathrm{H} 9 \mathrm{c} 2$ cardiomyocytes by NF- $\kappa \mathrm{B}$ dependent PUMA upregulation. Eur Rev Med Pharmacol Sci 17: 2323-2329, 2013.

6. Vimalraj S and Selvamurugan N: MicroRNAs expression and their regulatory networks during mesenchymal stem cells differentiation toward osteoblasts. Int J Biol Macromol 66: 194-202, 2014

7. Palmero EI, de Campos SG, Campos M, de Souza NC, Guerreiro ID, Carvalho AL and Marques MM: Mechanisms and role of microRNA deregulation in cancer onset and progression. Genet Mol Biol 34: 363-370, 2011.

8. Harries LW: MicroRNAs as Mediators of the ageing process. Genes (Basel) 5: 656-670, 2014.

9. Eshel O, Shirak A, Dor L, Band M,Zak T, Markovich-Gordon M, Chalifa-Caspi V, Feldmesser E, Weller JI, Seroussi E, et al: Identification of male-specific amh duplication, sexually differentially expressed genes and microRNAs at early embryonic development of Nile tilapia (Oreochromis niloticus). BMC Genomics 15: 774, 2014.

10. Feng Y, Liu J, Kang Y, He Y, Liang B, Yang P and Yu Z: MiR-19a acts as an oncogenic microRNA and is up-regulated in bladder cancer. J Exp Clin Cancer Res 33: 67, 2014.

11. Roff AN, Craig TJ, August A, Stellato C and Ishmael FT: MicroRNA-570-3p regulates HuR and cytokine expression in airway epithelial cells. Am J Clin Exp Immunol 3: 68-83, 2014.

12. Malizia AP and Wang DZ: MicroRNAs in cardiomyocyte development. Wiley Interdiscip Rev Syst Biol Med 3: 183-190, 2011.

13. Kang B, Hong J, Xiao J, Zhu X, Ni X, Zhang Y, He B and Wang Z: Involvement of miR-1 in the protective effect of hydrogen sulfide against cardiomyocyte apoptosis induced by ischemia/reperfusion. Mol Biol Rep 41: 6845-6853, 2014.

14. Pan Z, Sun X, Ren J, Li X, Gao X, Lu C, Zhang Y, Sun H, Wang Y, Wang H, et al: MiR-1 exacerbates cardiac ischemia-reperfusion injury in mouse models. PLoS One 7: e50515, 2012.

15. Lu JT, He W, Song SS and Wei W: Paeoniflorin inhibited the tumor invasion and metastasis in human hepatocellular carcinoma cells. Bratisl Lek Listy 115: 427-433, 2014.

16. Li JZ, Wu JH, Yu SY, Shao QR and Dong XM: Inhibitory effects of paeoniflorin on lysophosphatidylcholine-induced inflammatory factor production in human umbilical vein endothelial cells. Int J Mol Med 31: 493-497, 2013.

17. Kong P, Chi R, Zhang L, Wang N and Lu Y: Effects of paeoniflorin on tumor necrosis factor- $\alpha$-induced insulin resistance and changes of adipokines in 3T3-L1 adipocytes. Fitoterapia 91: 44-50, 2013. 
18. Wang K, Zhu L, Zhu X, Zhang K, Huang B, Zhang J, Zhang Y, Zhu L, Zhou B and Zhou F: Protective effect of paeoniflorin on A 325 -35-induced SH-SY5Y cell injury by preventing mitochondrial dysfunction. Cell Mol Neurobiol 34: 227-234, 2014.

19. Li JZ, Yu SY, Wu JH, Shao QR and Dong XM: Paeoniflorin protects myocardial cell from doxorubicin-induced apoptosis through inhibition of NADPH oxidase. Can J Physiol Pharmacol 90: 1569-1575, 2012.

20. Livak KJ and Schmittgen TD: Analysis of relative gene expression data using real-time quantitative PCR and the $2^{-\Delta \Delta C t}$ method. Methods 25: 402-408, 2001

21. Li J, Dong X, Wang Z and Wu J: MicroRNA-1 in cardiac diseases and cancers. Korean J Physiol Pharmacol 18: 359-363, 2014.

22. Yu XY, Song YH, Geng YJ, Lin QX, Shan ZX, Lin SG and $\mathrm{Li}$ Y: Glucose induces apoptosis of cardiomyocytes via microRNA-1 and IGF-1. Biochem Biophys Res Commun 376: 548-552, 2008.

23. Tang Y, Zheng J, Sun Y, Wu Z, Liu Z and Huang G: MicroRNA-1 regulates cardiomyocyte apoptosis by targeting Bcl-2. Int Heart J 50: 377-387, 2009.

24. Cha HJ, Kim OY, Lee GT, Lee KS, Lee JH, Park IC, Lee SJ, Kim YR, Ahn KJ, An IS, et al: Identification of ultraviolet $B$ radiation-induced microRNAs in normal human dermal papilla cells. Mol Med Rep 10: 1663-1670, 2014.
25. Fatemi N, Sanati MH, Shamsara M, Moayer F, Zavarehei MJ, Pouya A, Sayyahpour F, Ayat H and Gourabi H: TBHP-induced oxidative stress alters microRNAs expression in mouse testis. J Assist Reprod Genet 31: 1287-1293, 2014

26. Schmelzer C, Kitano M, Rimbach G, Niklowitz P, Menke T, Hosoe K and Döring F: Effects of ubiquinol-10 on microRNA-146a expression in vitro and in vivo. Mediators Inflamm 2009: 415437, 2009.

27. Simone NL, Soule BP,Ly D, Saleh AD, Savage JE, DegraffW, Cook J, Harris CC, Gius D and Mitchell JB: Ionizing radiation-induced oxidative stress alters miRNA expression. PLoS One 4: e6377, 2009.

28. Chen T, Ding G, Jin Z, Wagner MB and Yuan Z: Insulin ameliorates miR-1-induced injury in $\mathrm{H} 9 \mathrm{c} 2$ cells under oxidative stress via Akt activation. Mol Cell Biochem 369: 167-174, 2012.

29. Zhang S, Liu X, Bawa-Khalfe T, Lu LS, Lyu YL, Liu LF and Yeh ET: Identification of the molecular basis of doxorubicin-induced cardiotoxicity. Nat Med 18: 1639-1642, 2012.

30. Ma J, Wang Y, Zheng D, Wei M, Xu H and Peng T: Rac1 signalling mediates doxorubicin-induced cardiotoxicity through both reactive oxygen species-dependent and -independent pathways. Cardiovasc Res 97: 77-87, 2013.

31. Zhao Y, Zhou G, Wang J, Jia L, Zhang P, Li R, Shan L, Liu B, Song X, Liu S and Xiao X: Paeoniflorin protects against ANIT-induced cholestasis by ameliorating oxidative stress in rats. Food Chem Toxicol 58: 242-248, 2013. 